\title{
Investigation into mechanisms of deflagration-to-detonation using Direct Numerical Simulations
}

\author{
Joseph Adoghe ${ }^{1}$, Weiming Liu ${ }^{1}$, Jonathan Francis ${ }^{1}$ and Akinola Adeniyi ${ }^{1}$ \\ School of Engineering, University of Central Lancashire, \\ Preston, Lancashire, UK, PR1 2HE
}

\begin{abstract}
Detonation, a combustion phenomenon is a supersonic combustion wave which plays critical role in the theory and application of combustion. This work presents numerical investigation into indirect initiation of detonation using direct numerical simulations (DNS). The Adaptive Mesh Refinement in object-oriented C++ (AMROC) tool for parallel computations is applied in DNS. The combustion reactions take place in a shock tube and an enclosure with a tube respectively and are controlled by detailed chemical kinetics. The database produced by DNS accurately simulates the process of transition of deflagration to detonation (DDT), and investigates the influence of overpressure and kinetics on flame propagations during combustion processes. The numerical simulations showed the influence of pressure and kinetics to the transition of slow and fast flames and DDT during flame propagations. When the reaction rate is fast, DDT is achieved, but when slow, DDT will not occur and therefore, there will be no detonation and consequently no strong explosion. Exploring the influence of free radical $\mathrm{H}$ on flame propagation showed that the concentration of the reacting species decreased with flame speed increase for each propagation. Hence, the heat generated was very fast with a greater chance of DDT being triggered because flame speed increased.
\end{abstract}

\section{Nomenclature}

$\begin{array}{ll}\text { Abbreviations } & \text { Acronyms } \\ \text { SF } & \text { Slow flame } \\ \text { FF } & \text { Fast flame } \\ \text { DDT } & \text { Deflagration-to-Detonation Transition } \\ \text { DD } & \text { Direct Detonation } \\ \text { DNS } & \text { Direct Numerical Simulation } \\ \text { HRR } & \text { Heat Release Rate (Kw) } \\ \text { CFD } & \text { Computational Fluid Dynamics } \\ \text { FDS } & \text { Fire Dynamic Simulation } \\ \text { PMS } & \text { Premium motor spirit } \\ \text { AMROC } & \text { Adaptive Mesh Refinement in Object- } \\ & \text { Oriented C++ } \\ \text { Q } & \text { Heat }\end{array}$

\section{Introduction}

Explosions occasionally happen in industrial processes and in daily life. When they occur, they can produce big overpressures and associated fires which can usually make a great amount of damage and may be catastrophic. Explosions have posed one of the greatest threats to the oil and gas Industry (in areas of human losses, environmental damages, economic/financial losses coupled with property loss such as storage tank damage) [1]. A good understanding of the mechanisms and consequences of explosion is a key for designing fire safety management programmes to not only protect the assets of an organization, but also to ensure the safe evacuation of all the personnel present. Explosions could be classified as either mechanical explosion (e.g. caused by mechanical failure of a pressure vessel), or chemical explosion that is driven by chemical reactions. In a chemical explosion, rapid chemical reactions release a large amount of energy and therefore produce huge overpressure [2]. The reactions consist of various chemical chain reactions which are initiated when the fuel is broken down by heat. The chain reactions involve free hydrogen atoms, $\mathrm{H}$, hydroxyl free radicals, $/ \mathrm{OH}$, and free oxygen molecules and fuel is converted to vapour gaseous state by combustion [3]. Nonetheless, fuels such as solids and liquids can be converted to free radicals without being in vapour state. For example, during the combustion of hydrocarbons, carbon dioxide, water and heat are formed [4].

Besides, if there is fuel, oxidizer and energy in their appropriate amounts and the chemical chain reaction is not interrupted, the combustion process would continue [5]. The physical aspects of combustion play an important role. They comprise processes of heat transfer, movements of reactants and combustion products and mass transfer. The physical processes produce the circumstance and an environment to maintain the chemical reactions, while the reactions provide original forces to drive the physical processes [6]. Eventually, their interactions may result in various complex explosion modes. Although many researches were carried out in past years involving hydrogen and oxygen, liquid hydrogen, the study of hydrocarbon gases using mathematical programming [7], but this work will investigate detailed mechanisms of the explosions of hydrocarbon gases in petroleum storage tank farms using CFD simulations. This research work will particularly investigate the physicochemical mechanisms of petroleum gas explosions, and through the investigations, it is expected to reduce the explosion problems in the petroleum storage tank farms.

\section{Numerical Methods}

The combustion investigated in this work is controlled by the reacting Navier - Stokes equations of twodimension, 
$\frac{\partial \rho}{\partial t}+(u \cdot \nabla) \rho+\rho \nabla \cdot u=0$

$\rho \frac{\partial u}{\partial t}+\rho(u \cdot \nabla) u+\nabla p=\mu \nabla \cdot\left\{\nabla u+(\nabla u)^{T}\right\}$

$\rho \frac{\partial e}{\partial t}+\rho(u \cdot \nabla) e+p(\nabla \cdot u)=\nabla \cdot(\lambda \nabla T)+\Phi$

$\rho \frac{\partial Y_{i}}{\partial t}+\rho(u \cdot \nabla) Y_{i}=\nabla \cdot\left(\rho D_{i} \nabla Y_{i}\right)+\rho \omega_{i}$

where $u, \rho, T, p, e$ and $\Phi$ are, respectively, the velocity vector, density, temperature, pressure, internal energy, and dissipation function of the gaseous mixture, while $\lambda$ and $\mu$ are the conduction coefficient and viscosity of the gas mixture, respectively. The internal energy in (3) can be expressed by $e=c_{v} T$, where $c_{v}$ is the specific heat capacity at constant volume.

The equation (4) is a set of transport equations for reactions in which $Y_{i}$ is the fraction of concentration for the species $\mathrm{i}$ and $D_{i}$ is its diffusion coefficient. Because of $\sum Y_{i}=1$ and the continuity equation (1), $\sum\left[\nabla \cdot\left(\rho D_{i} \nabla Y_{i}\right)\right]=0$ is required. The term, $\omega_{i}$, in the equation is the reaction rate that will be determined by chemical kinetics.

The thermodynamical state equation is required to setup the relationship of the pressure, density and temperature

$p=\frac{\rho R T}{W}$

$W=\sum\left(\frac{Y_{i}}{W_{i}}\right)^{-1}$

where $R$ is the universal gas constant and $W$ is the molecular mass of the gas mixture which is calculated by (6). In equation (6), $W_{i}$ is the molecular mass of the species $i$.

Chemical kinetics: It is used to decide the reaction rate in the equation (4). The reaction rate can be calculated by

$\omega=k \prod c_{i}^{\alpha^{i}}$

where where $c$ is the concentration of reactant species and $\alpha$ is the reaction order. The reaction coefficient $k$ is controlled by the detailed chemical reactions. In this work we study into the reacting flows of hydrogen oxygen mixture and kinetics by $\mathrm{Li}$ et al [14] is employed.

Numerical schemes: The equations (1) - (6) are calculated by finite volume method on structured mesh, in which the Riemann problems are solved by high-resolution Godunov schemes [15].

Adaptive refinement of the mesh is applied to ensure the computational results being sufficiently accurate in the domain where the unknowns vary strongly. The adaptive refinement of mesh will generate a hierarchy of mesh. This hierarchical meshes are maintained and manupulated by the software, AMROC, which was developed by [16].

\section{Setup of numerical modelling}

Two cases are studied in this work. The first case study domain is a $2 \mathrm{D}$ rectangular box with dimension of $6 \mathrm{~cm} \times 5 \mathrm{~cm}$ and a tube with $2 \mathrm{~cm} \times 20 \mathrm{~cm}$ (see Figure 1 ). The second case study domain is a $2 \mathrm{D}$ shock tube of the size, $2 \mathrm{~cm} \times 40 \mathrm{~cm}$, only. These two case studies allow us investigating into DDT with different ignition stages. The domains are meshed by square elements and the initial element is $1 \mathrm{~mm}$ long and $1 \mathrm{~mm}$ wide. This initial mesh will be adaptively refined until the finest mesh resolution is reached to $0.025 \mathrm{~mm}$. It means that there are about 40 nodes in the zone of combustion reactions.

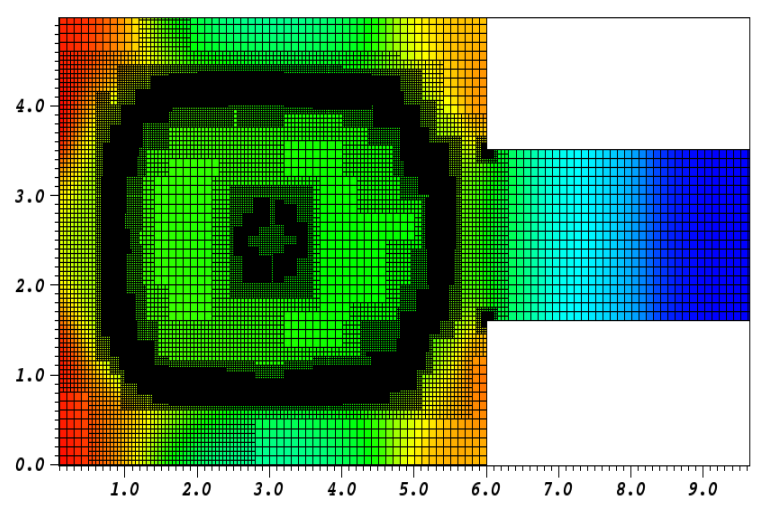

Fig. 1. Compuational Domain and Mesh

The simulated scenarios were mainly to investigate the influence of concentration and pressure on flame propagation during combustion processes. The reaction rates are dominant for processes of different combustion modes - slow flame (SF), fast flame (FF), deflagration-todetonation transition (DDT) and detonation. To simulate the reaction processes accurately, an integration time step is in the order of $10^{-8}$ second.

Three cases were studied, and their initial conditions are shown in Table 1. The "Hot" conditions are for igniting the reaction and in a small circular area of the radius of $2.5 \mathrm{~mm}$ at the box centre. The "Cool" conditions are in the other domain unreacted initially.

Moreover, on Table 1 below, the hot area is of very high temperature and pressure while the cool domain contains the premixed combustibe gas mixture which keeps constant the temperature and pressure, but the concentrations of chemical species comprising the combustible gas mixture are varied with different case studies. Additionally, on the hot area, the temperature is very high, and the concentrations of $\mathrm{H}_{2}$ 
and $\mathrm{O}_{2}$ are zero, therefore, no fuel $\left(\mathrm{H}_{2}\right)$, no oxygen $\left(\mathrm{O}_{2}\right)$. It should be noticed that normal chemical reaction takes maximum 2 species to react with 1 or 2 , but in enhanced reaction, it needs a third species for the reaction and the third-party role is not specific and it is to attack other molecules and not participating in the reaction, e.g., noble gases like $\mathrm{He}$ and $\mathrm{Ar}$,

$$
\mathrm{CH}_{3}+\mathrm{CH}_{3}\left(\mathrm{~N}_{2}\right)=\mathrm{C}_{2} \mathrm{H}_{6}+\mathrm{N}_{2}
$$

In the above example, $\mathrm{N}_{2}$ is the enhanced concentration because it is for a particular species in this reaction, but for the other normal reaction, $\mathrm{k}_{0}$ and $\mathrm{k}_{\infty}$ (infinity) are pressure independent and $\mathrm{M}$ dependent.

There are two kinds of boundary conditions, wall boundary conditions and outlet boundary conditions. On the walls, no-slip and adiabatic condions are applied and no-reflecting conditions are specified on the outlet.

Table 1. Initial Conditions using shock tube

\begin{tabular}{|c|c|c|c|c|c|c|c|c|}
\hline $\mathrm{C}$ & \multicolumn{2}{|c|}{ Tempt (K) } & \multicolumn{4}{|c|}{ Concentration (\%) } & \multicolumn{2}{|c|}{ Pressure } \\
\hline a & \multirow[t]{3}{*}{ Hot } & \multirow[t]{3}{*}{ Cool } & \multicolumn{2}{|c|}{ Hot } & \multicolumn{2}{|c|}{ Cool } & \multirow{3}{*}{$\begin{array}{r}\text { Hot } \\
\times 10^{5}\end{array}$} & \multirow{3}{*}{$\begin{array}{r}\text { Cool } \\
\times 10^{5}\end{array}$} \\
\hline$S$ & & & $\mathrm{H}_{2}$ & $\mathrm{O}_{2}$ & $\mathrm{H}_{2}$ & $\mathrm{O}_{2}$ & & \\
\hline$S$ & & & & & & & & \\
\hline 1 & 3,000 & 300 & 0.0 & 0.0 & 0.15 & 0.20 & 2 & 1 \\
\hline 2 & 3,000 & 300 & 0.0 & 0.0 & 0.15 & 0.20 & 5 & 1 \\
\hline 3 & 3,000 & 300 & 0.0 & 0.0 & 0.15 & 0.20 & 1 & 1 \\
\hline 4 & 2,800 & 300 & 0.0 & 0.0 & 0.68 & 0.17 & 2 & 1 \\
\hline 5 & 2,800 & 300 & 0.0 & 0.0 & 0.68 & 0.17 & 5 & 1 \\
\hline 6 & 2,800 & 300 & 0.0 & 0.0 & 0.68 & 0.17 & 1 & 1 \\
\hline
\end{tabular}

\section{Results and Discussions}

The results of the Two case senerios are presented and discussed in this section. The first case study simulates the first 3 cases of Table 1 above, while the second case study does all the 6 cases (see Tables 6 and 7 shown below).



Fig. 2. Concentration $\left(\mathrm{kg} / \mathrm{m}^{3}\right)$ of free radicals, $\mathrm{H}$ against flame speed $(\mathrm{m} / \mathrm{s})($ Cases 1, 2 3).
Table 2. Summary Concentrations of free radicals, $\mathrm{H}$

\begin{tabular}{|c|c|c|c|c|c|c|}
\hline $\mathrm{C}$ & Time range & Conc. range & \multicolumn{4}{|c|}{ Flame Speed $(\mathrm{m} / \mathrm{s})$} \\
\hline $\begin{array}{l}\mathrm{s} \\
\mathrm{e} \\
\mathrm{s}\end{array}$ & $\times 10^{-4}$ & $\times 10^{-4}$ & $\mathrm{SF}$ & FF & DDT & $\mathrm{DD}$ \\
\hline 1 & $1.0-2,2$ & $107.2-89.71$ & 7 & 300 & 470 & 2800 \\
\hline 2 & $0.9-2.65$ & $110.2-87.06$ & 10 & 250 & 470 & 2700 \\
\hline 3 & $1.95-6.70$ & $76.13-37.03$ & 8 & 270 & 480 & 2600 \\
\hline
\end{tabular}

Table 3. Concentration influence on flame development

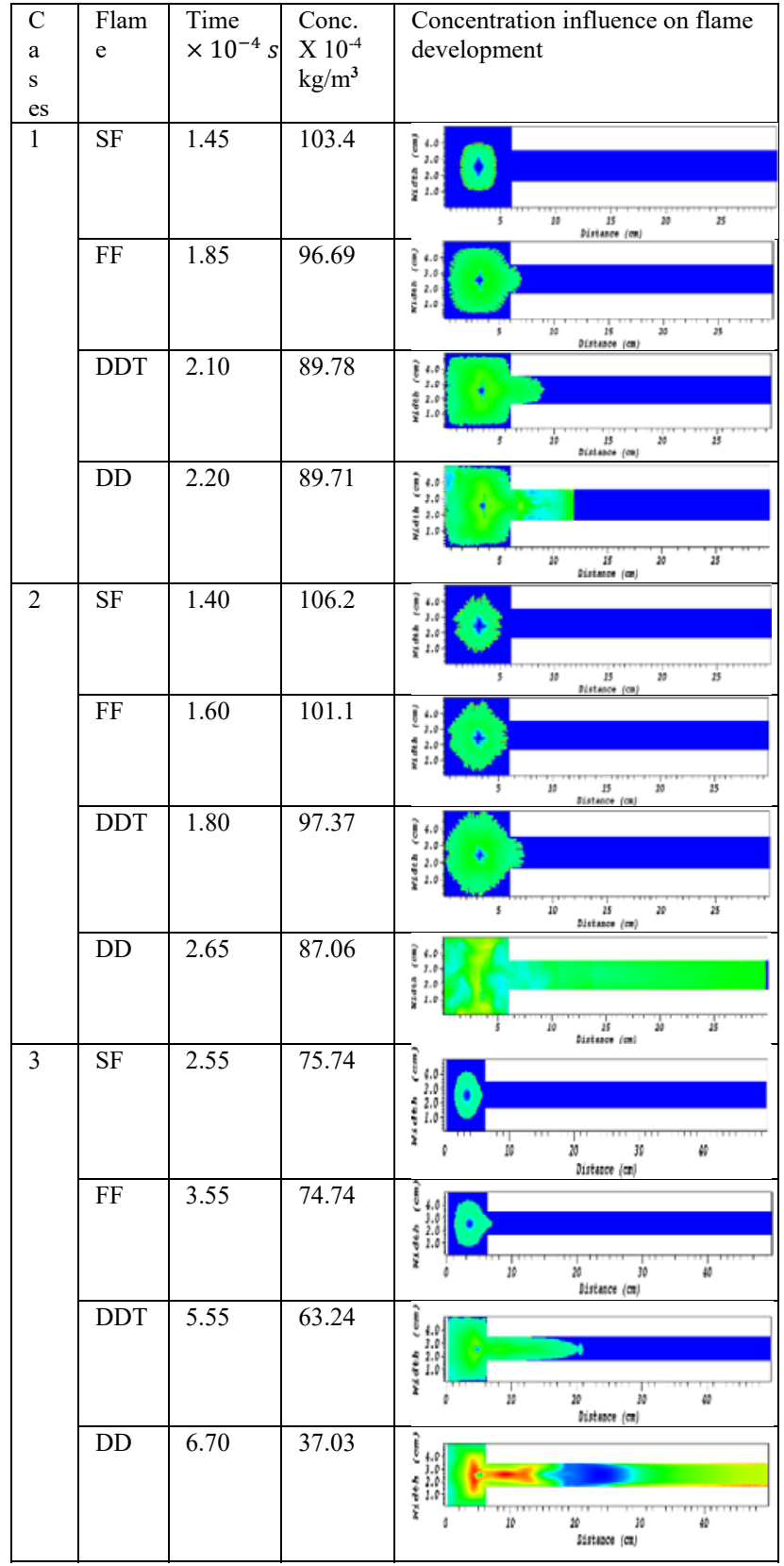


Table 4. Summary simulation results by pressure $(\mathrm{Pa})$

\begin{tabular}{|l|l|l|l|l|l|l|}
\hline $\mathrm{C}$ & Time & Pressure & \multicolumn{4}{|c|}{ Flame Speed (m/s) } \\
\cline { 4 - 7 } $\mathrm{a}$ & range & range $(\mathrm{Pa})$ & $\mathrm{SF}$ & $\mathrm{FF}$ & $\mathrm{DDT}$ & $\mathrm{DD}$ \\
$\mathrm{s}$ & $(\mathrm{sec})$ & $\mathrm{x} 10^{5}$ & & & & \\
$\mathrm{e}$ & $\times 10^{-4}$ & & & & & \\
$\mathrm{~s}$ & & & & & & \\
\hline 1 & $0.05-2.05$ & $1.008-8.77$ & 8 & 300 & 450 & 1330 \\
\hline 2 & $0.9-2.65$ & $1.979-28.30$ & 10 & 300 & 480 & 2700 \\
\hline 3 & $0.25-6.65$ & $1.001-28.98$ & 7 & 260 & 490 & 2600 \\
\hline
\end{tabular}

Table 5. Pressure influence on flame development

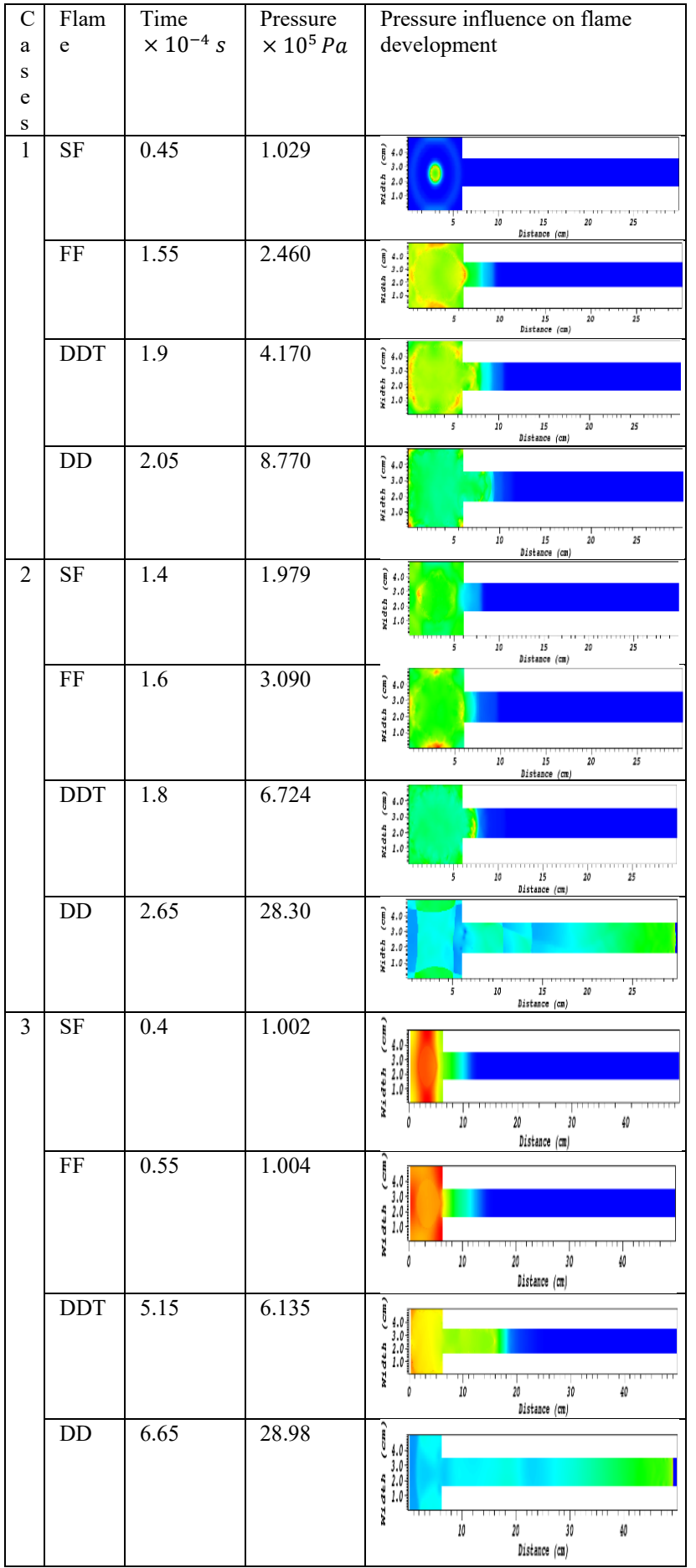

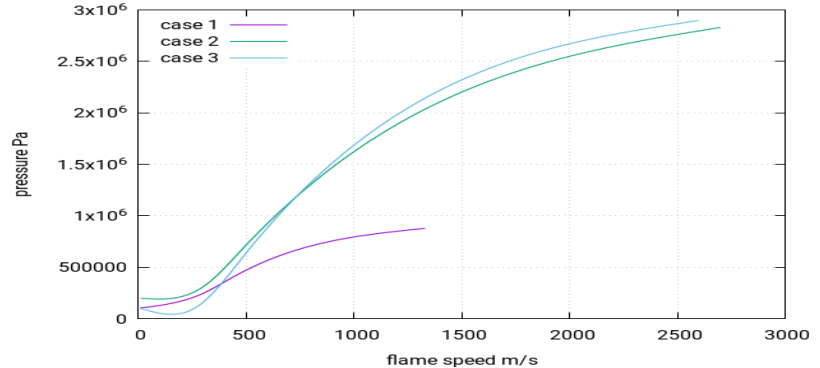

Fig. 3. Pressure against flame speed (m/s) for cases 1, 2, 3 .

First let us explore the results of first case study, i.e., first three cases of Table 1 above, of the domain of Figure 1 and their results are showed in Figure 2, Figure 3, and Tables $2-5$.

From Tables 2, 3 and Figure 2 above, it was observed that as the concentration of the reacting species for each case decreases, the combustion equally decreases and the flame velocity increases, but for DDT, explosion is formed because the flame is not uniform.

From Tables 4, 5 and Figure 3 above, one can see as the pressure increases, the flame velocity increases. Moreover, DDT always starts at a point and then spreads. When the pressure is very high, it implies that the shock wave is equally high. Also, it was noticed that there was a drastical drop in pressure in case studies 2 and 3 during detonation from $4.889 \times 10^{6} \mathrm{~Pa}$ to $2.830 \times 10^{6} \mathrm{~Pa}$ and from $8.308 \times$ $10^{6} \mathrm{~Pa}$ to $2.898 \times 10^{6} \mathrm{~Pa}$, and terminates at 0.000265 seconds and 0.00067 seconds for cases 2 and 3 respectively, and this is due to high shock wave that went out of the tube.

Furthermore, now we move on to explore the results of the case study two. Their results are presented in Table 6 and 7.

The simulation picture colours in Table 6 below show the blue locations represent minimum or zero concentration; the yellow indicate between blue and green; the green stands for concentration more than zero but less than yellow; and the red shows maximum or largest volume of concentration. In addition, the flame fronts of the pictures of the different combustion forms formed showed that for detonation, the flame front is flat/sharp because it is a very fast detonation and boundary layer effect is very small and flame front move along, but for DDT, its flame front is curved due to its instability, boundary layer effect and numerical error due to computer capability; and for laminar flame, it is very viscous. Furthermore, in each case study of case studies 1-3 of table 6 above, it portrayed that as the concentration increases, the temperature decreases, thereby generating low heat leading to slow flame production.

However, in each case study of case studies $4-6$, as the concentration of the reacting species increases, the temperature increases, and more heat release rate is produced. Hence, more DDT and detonation are possibly formed as more products are produced. 
Table 6. Concentration influence on flame development

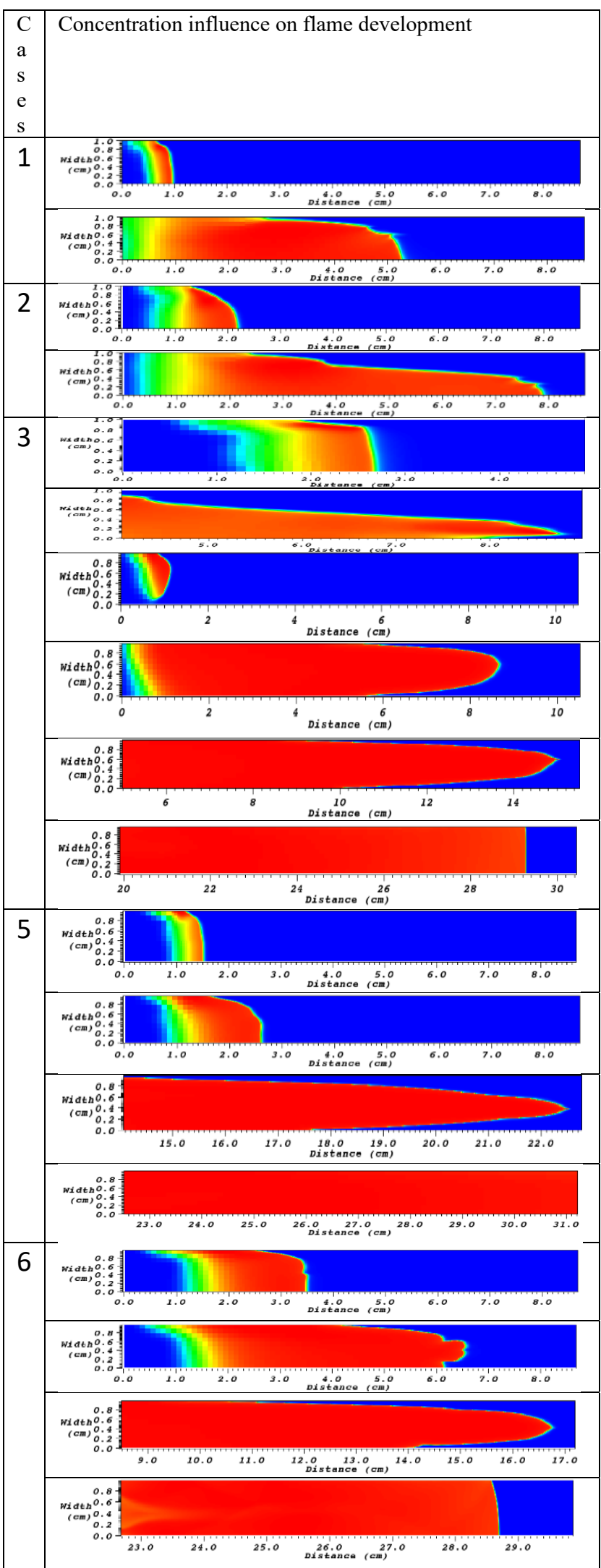

Table 7. Pressure (Pa) influence on flame development

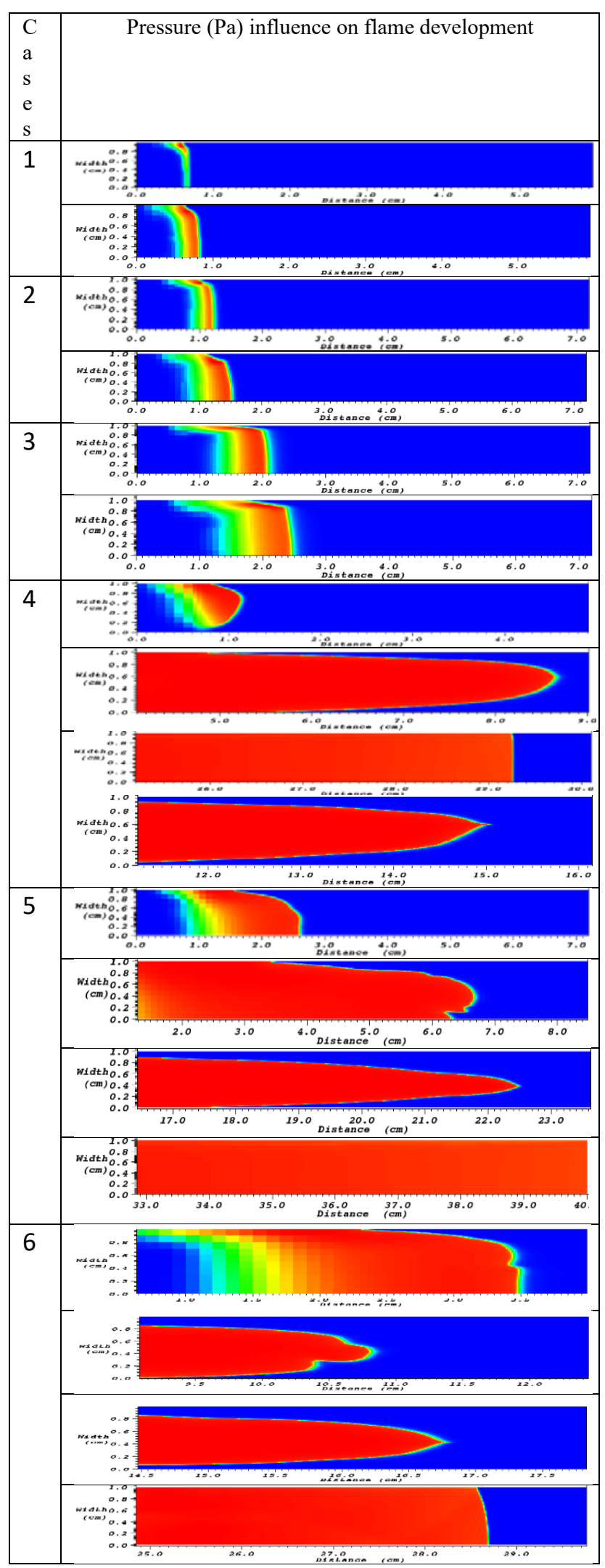


From Table 7 above, it showed that as the pressure increases and the temperature increases, consequently, the reaction rate of the combustion process increases and DDT and detonation are finally obtained.

\section{Conclusion}

This research work investigated the mechanisms and consequences of deflagration to detonation transition processes using CFD Simulations. The mechanisms and consequences of explosions were studied by chemical reaction mechanisms of chemical combustion reactions of fuels using a shock tube and a vented tube respectively, and critically analysing the processes of transition of deflagration to detonation. Moreover, the purpose of this research work presently is to investigate the influence of overpressure and kinetics on various flame propagations during chemical combustion reaction processes. Furthermore, the numerical simulations show the influence of pressure and kinetics on flame propagation ranging from slow flame, fast flame and DDT to detonation. Usually, different ignition sources will give different detonation and different processes have different DDT results. If pressure is drastically reduced, DDT will not take place. When the reaction rate is fast, DDT will be achieved, and more detonation are formed thereby producing explosion.

Using free radical, $\mathrm{H}$ to judge the influence of concentration on flame propagation, it shows that as the concentration of the reacting species decreases, temperature and pressure increase, then heat generated will be very fast and more DDT and more detonation will be formed for case studies 4 , 5 and 6 , but using product reaction, $\mathrm{H}_{2} \mathrm{O}$, its concentration increases with increase in temperature and pressure for case studies 4, 5 and 6, thereby generating more heat, more products and consequently more DDT and more detonation leading to more explosions. To this background, the AMROC simulation results obtained in this research work showed that when reaction rate is fast, DDT will be achieved and more explosions result, but if reaction rate is slow, DDT and detonation will not be formed for reactions in case studies $1-3$ and thus, there will be no explosions. As expected, pressure varies proportionally with speed, and thus, if it is a slow flame, it is very easy to fight, but fast flame is more difficult to fight. In this research, vent is created in the tube, and if there is no vent, there will be no explosion and this is because the burnt wave is deflagration and not detonation. Hence, when vent is created in the tube, DDT will occur and consequently detonation is achieved and vent explosion will take place.

Moreover, this is to predict to a certain percentage of accuracy of explosion protection. Thus, detonation is so much very fast that it spreads greatly thereby leading to vent explosion and it terminates at 0.000265 seconds and 0.00067 seconds and with a pressure of $2.830 \times 10^{6} \mathrm{~Pa}$ and $2.898 \times 10^{6} \mathrm{~Pa}$ for case studies 2 and 3 respectively.

\section{Acknowledgments}

This project is funded by the Petroleum Technology Development Fund (PTDF), Federal Republic of Nigeria which is sincerely acknowledged. The authors would like to appreciate the University of Central Lancashire, Preston, UK for providing the AMROC tool within the framework of OPENSuse that gave a unique basis for this study.

\section{References}

1. M. H. Abbasi, E. Benhela and A. Ahmadl, "Designing an Optimal Safe Layout for a Fuel Storage Tanks Farm: Case study of Jaipur Oil Depot," International Journal of Chemical, Molecular, NuclearMaterials and Metallurgical Engineering, vol. 8, no. 2, pp. 147-155, 2014.

2. Society of Fire Protection Engineering, SFPE Handbook of Fire Protection Engineering, 18th edition ed., New York: Springer Publishers, 2016.

3. L. H. Ferguson and C. A.Janicak, Fundamentals of Fire Protection for the Safety Professional, First edition ed., Maryland: Government Institutes, An imprint of The Scarecrow Press Inc, 2005.

4. I. Glassman, R. A. Yetter and N. G. Glumac, Combustion, London: Elsier Inc., 2015.

5. P. E. Ross, "Fire Prevention: A guide to protecting employees and property," Journal of professional safety, vol. 56, no. 10, pp. 62-69, 2011.

6. E. Ufuah and C. G. Bailey, "Flame radiation characteristics of open hydrocarbon pool fires," in Proceedings of the World congress on Engineering, London, UK, 2011.

7. Z. Ruming, N. Baisheg, H. Xueqiu, W. Chao, Z. Caihong, D. Linchao, L. Qian, L. Xinna and L. Hailong, "Different gas explosion mechanisms and explosion suppression techniques," Journal of Procedia Engineering, vol. XVI, pp. 14671472, 2011.

8. J. F. Wendt, Computational Fluid Dynamics AN INTRODUCTION, 3rd edition ed., Belgium: Springer-Verlag Heidelberg, 2009.

9. G. H. Yeoh and K. K. Yeun, Computational Fluid Dynamics in Fire Engineering: Theory, Modeling and Practice, London: Elsier Academic Press, 2010.

10. A. A. Date, Introduction to computational fluid dynamics, New York: Cambridge University Press, 2009.

11. B. N. Chetverushkin and A. V. Gulin, "Explicit schemes and numerical simulation using ultrahighperformance computer systems," Journal of 
Doklady Mathematics, vol. 86, no. 2, pp. 681-683, 2012.

12.X. Feng, Y. He and P. Huang, "A Stabilized implicit fractional-step method for the timedependent Navier-Stokes equations using equalorder pairs," Journal of Mathematical Analysis and Applications, vol. 392, no. 2, pp. 209-224, 2012.

13 H. J. F.-S. Lien and E. Yee, "A new adaptive mesh refinement data structure with an application to detonation," Journal of computational physics, vol. 229, no. 23, pp. 8981-8993, 2010.

14.Li J, Zhao Z, Kazakov A and Dryer F L, An updated comprehensive kinetic model of hydrogen combustion, International Journal of Chemical Kinetics, Vol. 36 (2004), pp 566 - 575

15.LeVeque,R J, Finite volume methods for hyperbolic problems, Cambridge University Press, 2003, Cambridge

16.Deiterding R, Parallel adaptive simulation of multidimensional detonation structure, $\mathrm{PhD}$ Thesis, BTU Cottbus, 2003. 